\title{
IL PUNTO SU SEBASTIJAN KRELJ
}

Come Sebastianus Krelo Austriacus si era iscritto all'Accademia di Jena nel 1557 colui che oggi viene considerato uno dei protagonisti di spicco dell'epopea protestante slovena. Sono ormai parecchi anni che, nel celebrare l'anniversario del 1584 nella cultura e storia slovena (anno della pubblicazione della traduzione della Bibbia ad opera di Jurij Dalmatin e della prima grammatica slovena, scritta in latino, Articae horulae di Adam Bohorič), non si contano ormai più libri, saggi, articoli, convegni e commemorazioni varie così in Slovenia come all'estero ed in particolare a Derendingen, oggi periferia della città di Tübingen, che diventò la seconda patria di colui che viene considerato il padre della letteratura slovena, cioè Primož Trubar. E nella vicina città di Urach aveva sede l'importantissima tipografia dei protestanti sloveni e non: basterà ricordare che qui in soli cinque anni vennero alla stampa ben 50 tra pubblicazioni in sloveno, croato, glagolitico, italiano ecc. Ma queste dovrebbero essere cose abbastanza risapute. Meno conosciuta e, oseremmo dire quasi un po' trascurata in questa messe di commemorazioni, appare l'opera e l'importanza di uno dei protagonisti del protestantesimo sloveno, appunto Sebastijan Krelj. ${ }^{1}$

\section{CENNI BIOGRAFICI}

Krelj era nato a Vipava (Vipacco) nel 1538. Non sappiamo molto dei suoi primi studi. Lo ritroviamo come già detto a Jena dove ottiene il titolo di "magister". Qui è allievo, discepolo e infine amico di Matija Vlačić Illyricus (Mattia Flacio Illirico). Krelj ne diventa anche l'accompagnatore. Durante le sue frequenti visite a Ljubljana si fa apprezzare dal Trubar che lo nomina suo aiutante. E dopo la partenza definitiva del Trubar per la Germania nel 1565, il Krelj, malgrado abbia soltanto 27 an$\mathrm{ni}$, viene nominato primo predicatore ovvero sovrintendente della comunità protestante di Ljubljana. Già minato dalla tuberculosi, muore però soltanto due anni dopo, nel 1567. E questa prematura scomparsa sarà gravida di conseguenze per la diffusione delle sue idee come per la conoscenza delle sue opere, naturalmente non molte.

\footnotetext{
Nella sua Storia della letteratura slovena (con un profilo di letteratura serbo-lusaziana (Milano 1961) B. Meriggi dedica a S. Krelj scarne 18 righe nel capitolo Gli altri autori protestanti, pp. 32-33. Esauriente è invece la sintesi della sua biografia e opere nello Slovenski biografski leksikon I (Ljubljana $1925-32)$, pp. 565-567, corredato da una buona bibliografia. Nel più recente Primorski slovenski hiografski leksikon 8 (Gorica 1982), pp. 198-199 la presentazione del Krelj appare più concisa e stringata.
} 


\section{RAPPORTI VLAC̆IĆ-KRELJ}

Decisivo e fondamentale fu l'incontro a Jena del giovane Krelj con il grande istriano Matija Vlačić Illyricus (1520-1575), nativo di Labin (Albona). ${ }^{2}$ Il Flacio, staccatosi da Filippo Melantone, che rappresentava l'indirizzo più moderato del luteranesimo, fu l'ispiratore e grande realizzatore di quella monumentale storia ecclesiatica ispirata alla dottrina luterana che è conosciuta con il titolo Le Centurie di Magdeburgo. Il Flacio presiedeva un gruppo di studiosi che vennero poi chiamati i Centuriatori di Magdeburgo. Le prime tre centurie (suddivisione della materia per secoli) uscivano in un sol tomo nel 1559 , la tredicesima e ultima nel 1574 . Nel primo tomo era collocata una prefazione generale a tutta l'opera. Vi veniva esposta la vera fede cristiana, insegnata da Cristo e diffusa dagli apostoli e in tutto corrispondente a quella che ora insegnava il luteranesimo. Il vero protagonista dell'opera dei Centuriatori era però il papato in quanto sarebbe stato responsabile dell' oscuramento che per secoli aveva gravato sulla cristianità: nel papato veniva ravvisata la bestia dell'Apocalisse, l'Anticristo. ${ }^{3}$

Il Krelj assorbì con entusiasmo le idee e i concetti del maestro e amico Vlačić. E questo suo stretto legame con il Vlačić è sempre stato oggetto di discussione tra gli Sloveni e i Croati. Riassunto sinteticamente il discorso è questo: gli Sloveni ammettono l'innegabile influsso del Vlačić sul Krelj, ma ascrivono certe peculiarità linguistiche che trovano riscontro soltanto nel Krelj e non negli altri autori protestanti piuttosto che all'influsso del croato, alla conoscenza che il Krelj, nativo di Vipava, aveva dei vicini dialetti carsici mediani. I Croati invece sottolineano l'influsso inconfutabile del Flacio sulle idee e sull'opera del Krelj e nell'opera Otročja biblija (Bibbia per ragazzi), oggi attribuita al Krelj almeno per la parte slovena, indicano il Flacio almeno come coautore. Il fatto si spiega perchè l'opera fu pubblicata senza l'indicazione dell'autore o compilatore.

\section{RAPPORTO TRUBAR -KRELJ}

Il rapporto tra il "vecchio" Trubar e il "giovane" Krelj appare quanto meno singolare. Bisogna innanzitutto scindere i loro rapporti dalle loro idee nel campo linguistico e soprattutto ortografico. Il Trubar stesso aveva caldamente appoggiato e proposto la candidatura del Krelj a suo vice e con la sua partenza per la Germania ne aveva indirettamente favorito la nomina a sovrintendente della comunità luterana lubianese. Nessuna delle fonti a nostra conoscenza smentisce la loro ottima intesa a livello personale. Eppure, fin dalla sua prima opera importante, la Bibbia per ragaz-

2 L'opera più completa dedicata al grande istriano è Matija Vlačic Ilirik di M. Mirković (JAZU, Zagreb 1960) di ben 562 pagine e 44 appendici. Non sarà perciò inutile citare anche le pp. relative al Krelj: 36, 195, 223, 406, 451-453, 455, 457, 458, 462, 474, 476.

3 Si veda a questo proposito G. Cozzi, Storici del medioevo nell'età moderna (Centuriatori di Magdeburgo, C. Baronio, P. Sarpi, R. Simon) (Ed. CI.UEC, Venezia 1966). Per i centuriatori e il Flacio sono particolarmente interessanti le pp. $4-8$. 
zi, il Krelj aveva preso le distanze dagli insegnamenti linguistici del suo amicoprotettore Trubar. Era subito entrato in netta opposizione con lui. Nulla sappiamo delle reazioni del "grande vecchio". Resta però il fatto che nelle sue opere posteriori lo stesso Trubar tenne in considerazione le riforme ortografiche già attuate dal Krelj. E anche nella stesura definitiva della traduzione della Bibbia del Dalmatin e nella grammatica del Bohorič è stato riscontrato un influsso talmente notevole dell'ortografia del Krelj che, secondo alcuni studiosi sloveni, la scrittura slovena che conosciamo con il nome di "bohoričica" dovrebbe in realtà chiamarsi "kreljica". ${ }^{4}$

\section{OPERE}

Le opere del Krelj non sono numerose e la cosa non può sorprendere se consideriamo la sua prematura scomparsa a soli 29 anni. Fu lo stesso Vlačić a ispirare la sua prima opera e cioè Christlich bedencken (...) pubblicata a Eisleben nel $1562 .{ }^{5}$ L'opera fa parte di quelli scritti fortemente polemici che accompagnarono la polemica tra Flacio Illirico e Just Meni. Il Krelj è inoltre autore di 11 canti ecclesiatici di cui 5 sembrano originali mentre gli altri 6 sono traduzioni ispirate da modelli tedeschi. ${ }^{6}$

Nel 1563 il Krelj aveva inviato al barone Ungand ad Urach una Summe christlicher Lehre che però non fu mai stampata. Sembra però assodato che quest'opera fosse il preannuncio di quella Otročja biblija che, grazie all'appoggio del Flacio, venne pubblicata a Regensburg nel 1566, priva però dell'indicazione del suo autore oppure compilatore. Oggi le parti riguardanti lo sloveno sono unanimemente attribuite a Sebastijan Krelj, mentre resta dubbia la paternità delle parti in croato ovvero nelle altre lingue. La $O B$ non è infatti un'opera solamente slovena. Comprende cioè l'abecedario sloveno (lettere maiuscole e minuscole slovene, i loro nomi sloveni, le vocali, le sillabe, un dizionarietto latino-sloveno, un detto latino e quattro sloveni) a cui seguono i catechismi in sloveno, croato, tedesco, latino e italiano. Troviamo poi le norme di vita e una polemica Antithesis. Si arriva alla conclusione con un canto del Trubar e uno del Krelj. ${ }^{7}$

4 Si cfr. SBL I, op. cit., p. 567.

5 Ibidem, p. 566. Il titolo completo è Christlich bedencken, ob und sie fern sein iglicher christ, die rotten und secten, auch mit der that, zu richten, und zu anathematiziren, schuldig sey.

6 Si veda PSBL, op. cit., p. 198, compilatore M. Jevnikar.

7 Il Kidrič aveva presentato la $O B$ nel saggio $O$ sumljivih naslovih knjig $v$ z.godovini južnoslovanskega pismenstva reformacijske dobe (Titoli sospetti di libri nella storia della letteratura slava meridionale) in "Slavia" I (Praga 1922-1923), pp. 360-378 e in particolare nel capitoletto Catechesis slavica Crellii, pp. 365-367. Tra i libri di testo della scuola protestante di Lubiana viene infatti citata nel luglio 1575 una "Catechesis Sebastiani Crellii, quae continuet prima et praecipua nuda sex capita religionis christianae" e per un'altra classe una "Sebastiani Crellii Slavica quoque Catechesis" (p. 365). Si suppose perciò l'essistenza di un'opera perduta del Krelj ovvero un Catechismo. Il Kidrič però arriva alla conclusione che il citato Catechismo è in realtà la $O B$. (p. 567).

F. Kidrič analizzò attentamente la $O B$ in "ČJKZ" IV (Ljubljana 1924), pp. 121-125 (riedizione nei citati Izhrani spisi I, pp. 127-131) grazie all'aiuto di N. B. Jopson che da Londra gli inviò la descrizione accurata di tutta l'opera con la trascrizione esatta delle parti slovene e croate.

M. Mirković, op. cit., dedica ai lavori per la $O B$ le pp. 218-219 e 405.

Recentissimo è infine il saggio di M. Orožen, Kreljeva Otročja Biblija che accompagna la riedizione della $O B$ nella serie "Monumenta litterarum slovenicarum" 20 (Ljubljana 1987), pp. 139-157. La copia del reprint è quella romana. 
Pur essendo oggi considerata soprattutto un'opera del Krelj, la $O B$ rimane comunque una pubblicazione dei protestanti slavi meridionali, un'opera concepita a scopi soprattutto didattici. Era uno dei libri di testo della scuola protestante slovena. di Ljubljana. Secondo il Kidrič la parte del catechismo veniva adoperata nella I sezione della I classe, quella slovena nella II e quella tedesca nella terza sezione. Il dizionarietto latino-sloveno del Krelj insieme alla Nomenclatura del Bohorič venivano usati soprattutto come preparazione alla conoscenza dello sloveno. ${ }^{8}$

Il 30 ottobre 1589 a Ljubljana uscì l'ukaz: i pastori e gli insegnanti protestanti dovevano abbandonare quello stesso giorno Ljubljana e nei tre giorni successivi i territori austriaci. ${ }^{9}$ Segui la ben nota distruzione delle chiese e anche cimiteri protestanti, i libri protestanti vennero messi al rogo ecc. Ed è probabilmente per questa ragione che gli unici esemplari conosciuti della $O B$ si trovano oggi soltanto a Roma, Ulm e Londra. ${ }^{10}$

Diversa fortuna ebbe invece la seconda e purtroppo ultima opera del Krelj e cioè Postila slovenska (La postilla slovena). Se la $O B$ può considerarsi abbastanza originale nella composizione, la Postila slovenska è invece dichiaratamente la traduzione, anche se parziale e libera, della famosa postilla di J. Spangenberg che, pubblicata per la prima volta negli anni $1542-1544$, ebbe moltissime riedizioni e fu tradotta anche in latino. E noto che la grande fortuna e diffusione della Postilla dello Spangenberg si dovevano alla sua forma di dialogo e alla facile comprensione. Lo scopo era la spiegazione del Vangelo e dichiaratamente anche la propaganda della nuova fede in lotta con il cattolicesimo. ${ }^{11}$

La Postilla dello Spangenberg si compone di cinque parti. Nel 1567 il Krelj curò la traduzione in sloveno della sola parte invernale, ma benchè avesse parzialmente tradotto altre due parti, non potè terminarle. Se ne curò poi J. Jurišić (Georgius Jurishitzh) ma per la stampa bisogno aspettare altri dieci anni. Questa seconda edizione della Postila viene così datata 1587. Benchè allo Jurišić fosse stato espressamente ordinato di attenersi scrupolosamente alla riforma ortografica attuata dal Krelj, questa seconda edizione della Postila presenta notevoli differenze rispetto alla prima. Resta però difficile da stabilire se le modifiche siano dipese o dallo Jurišić o dal Bohorič o, al limite, dal tipografo o dal suo correttore. È infatti notorio lo strapotere dei tipografi dell'epoca in Germania.

J. Jurišić era croato, pertanto i numerosi croatismi nelle parti da lui curate per la Postila non sorprendono. Tutti e due gli autori erano inoltre profondi conoscitori della letteratura cirillica e glagolitica. Anzi, nel rapporto che il Krelj ha con il cirilli-

8 Cfr. Kidrič, Zgodovina slovenskega slovstva. Od začetkov do Zoisove smrti (Ljubljana 1929-1938), e dello stesso Slovenske knjige v protestantski stanovski šoli.v Ljubljani 15.63-1598 in "CJKZ" IV (Ljubljana 1924), pp. 130-139, ripubblicato nel già citato Izbrani spisi I, pp. 132-142.

9 Cfr. F. Kidrič, Zgodovina..., op. cit., p. 88.

10 Quest'ultimo esemplare forni al Kidrič la possibilità di descrivere la $O B$, vedi nota n. 7 .

11 Per l'importanza delle traduzioni delle postille in sloveno si veda soprattutto V. Oblak, Protestantske postile v slovenskem prevodu in "LMS" (Letopis Matice slovenske) (Ljubljana 1894), pp. 202-219. 
co potremmo già cogliere i primi accenni a quel movimento che andrà sotto il nome di panslavismo. Il Krelj infatti ravvisa nella bella scrittura cirillica, lepo čurilsko pismo, qualcosa di prettamente slavo mentre il realista pratico Primož Trubar non ne coglie affatto degli elementi nazional-esaltanti. ${ }^{12}$

\section{LA QUESTIONE DELL'ALFABETO}

L'analisi della lingua del Krelj ci viene offerta in maniera quanto mai esauriente dallo studioso sloveno J. Rigler. ${ }^{13}$ Egli sottolinea che il Krelj non potè oppure non osò rompere del tutto con la tradizione linguistica già istituzionalizzata dal Trubar, cioè con quella che egli stesso definì Gospod Truberiova Kranschina "il dialetto carniolo del signor Trubar". Tentò così di prendere almeno le dovute distanze dalle posizioni soprattutto ortografiche adottate dall'amico. Si avvicinò per quanto possibile alla lingua della letteratura così glagolitica che cirillica, laddove almeno queste due non apparivano in netto contrasto con le pecularità linguistiche dello sloveno. In un certo senso difese le proprietà e le caratteristiche dei dialetti sloveni "decentrati" rispetto a quelli sloveni "centrali". Il Krelj non si potrebbe certo definire "il primo sloveno patriota illirico", definizione che invece va a pennello per il Trubar che, per primo, difese l'individualità dello sloveno nei confronti delle altre lingue slave meridionali. ${ }^{14}$

Resta però il fatto che molte innovazioni ortografiche apportate dal Krelj, almeno per l'epoca, non ebbero successo, anche se poi si affermarono e furono istituzionalizzate nel corso dei secoli. Una delle cause preponderanti di questo insuccesso va certamente ascritta a una certa incoerenza e instabilità che le contraddistinguono. Valga per tutte l'esempio della trascrizione della jat paleoslava (ě, e speciale) che il Krelj propone per lo sloveno.

L'esito più usuale e frequente è la e normale: cfr. beseda 'parola', bolezen 'malattia', človek 'uomo', tutti e tre termini molto comuni. Fra quelli meno frequenti va ricordato pobegniti 'fuggire'. Sempre l'esito $e$ normale, ma soltanto nella maggioranza dei casi in cel 'intero', lep 'bello' e umreti 'morire'.

12 Cfr. F. Kidrič, Zgodovina..., op. cit., p. 86.

13 In Začetki slovenskega knjižnega jezika (Ljubljana 1986) e precisamente nei due saggi Kreljev jezik; pp. 117-141 e Razvoj bohoričice - Pri Krelju, pp. 219-222:

J. Rigler si occupa di S. Krelj anche nell'opera Razprave o slovenskem jeziku (a cura di F. Jakopin) (Ljubljana 1986) e precisamente alle pp. $24-25,30-38,47,51,59-64,223-226$.

Da ultima $\mathrm{M}$. Orožen analizza la concezione linguistica del Krelj nel contributo Kreljev jezikovni koncept in "XXIII. seminar slovenskega jezika, literature in kulture". (Ljubljana 1987), pp. 19-40.

14 Tanto che una monografia dedicata al Trubar, opera di J. Humar, porta il titolo Primož Trubar rodoljub ilirski (Koper 1980).

Citando il Kidrič (Zgodovina..., op. cit. pp. 33-34) J. Rigler, Kreljev jezik, op. cit., p. 117 defïnisce il Krelj "il nostro primo illirista", ciò̀ di tendenze illiriste e non sloveno-illiriste, cosa che invece vale per il Trubar. 
Però la jat è transcritta come é prevalentemente nei termini réć 'cosa', svét 'mondo', léto 'anno'. Un caso a sé è rappresentato dai termini greh 'peccato' e mesto 'luogo, posto, città'. Infatti nella $O B$ appaiono con $e$ nella $P S$ con é.

Terzo esito della jat nelle opere del Krelj è ei e in particolare nei casi obliqui del pronome ta 'costui': cfr. teih 'di questi', teim 'a questi' e steim 'con costui'. E inoltre nel locativo plurale se accentato: očeih 'negli occhi', dneih 'nei giorni' e Lüdeih 'nelle persone'. Nello sloveno odierno tutti e tre gli esiti possibili della jat paleoslava confluiscono in $e$ normale. Il Krelj però aveva già usato indifferentemente e molto incoerentemente tutte e tre le soluzioni possibili della jat nel presente negativo dell'ausiliare che oggi suona ne imeti, nimam 'non avere, non ho'. Si cfr. neima e néma 'non ha', nemaio 'non hanno'. Ma nella PS era già ricorso all'odierna $i$, si veda nima 'non ha' e nimajo 'non hanno'.

Sempre in campo fonetico destano particolare curiosità alcuni termini che sono contraddistinti dalla cosiddetta seconda palatalizzazione paleoslava che notoriamente in sloveno ha lasciato ben poche tracce. Nelle opere del Krelj queste appaiono ben più numerose. Troviamo così Junaci per Junaki 'gli eroi', proroci per preroki 'i profeti' ed anche Turci per Turki.'i Turchi'.

Inoltre per il pronome personale di prima persona "io", il Krelj usa iest, ias per l'odierno jaz, ma nella $P S$ troveremo anche $j a$ che non può che essere un croatismo. Indubbi croatismi sono pure stabom per s teboj 'con te' e pastirom per $s$ pastirjem 'con il pastore', quest'ultimo esempio di uso dello strumentale senza la preposizione.

Tutti questi esempi, pur se non numerosi, ci appaiono comunque probanti nel problematico o forse impossibile tentativo di chiarificazione del rapporto che lega la lingua del Krelj a quella croata.

\section{IL LESSICO KRELJANO CON PARTICOLARE RIFERIMENTO AI PRESTITI ROMANZI}

Gli storici letterari sloveni amano affermare nelle loro opere che, a differenza degli altri autori protestanti, il Krelj ha tentato di arginare la penetrazione dei prestiti germanici nel lessico sloveno favorendo piuttosto la penetrazione dei prestiti romanzi ovvero italianismi. Alla luce di un' analisi più attenta questa certezza, ormai diventata quasi un assioma, non appare più tale e va quanto meno ridimensionata. La convinzione era stata suffragata soprattutto dal fatto che il Krelj era nato in una provincia "decentrata" rispetto alle altre "centrali" dei suoi colleghi protestanti. Ed era l'unica provincia in stretto contatto con il mondo romanzo. Al Krelj si attribuisce inoltre così la conoscenza dell'italiano come del friulano. E va qui detto per inciso che negli studi degli ultimi anni si va chiaramente delineando l'importante funzione mediatrice operata dal friulano nel complesso processo di penetrazione dei presti- 
ti romanzi nello sloveno letterario, spesso e comunque prevalentemente attraverso i dialetti carsici. ${ }^{15}$ Se infatti oggi il dialetto di Vipava fa parte del cosiddetto gruppo dei dialetti sloveni interni (notranjsko), ai tempi del Krelj vi si parlava un dialetto con delle spiccate caratteristiche dei dialetti carsici, a meno che non fosse un dialetto carsico vero e proprio. Stanno a dimostrarlo gli stessi esiti fonetici per i quali opta il Krelj, esiti che sono in netto contrasto con quelli del Trubar e degli altri autori protestanti, ma trovano tutti riscontro nei vicini dialetti carsici e spesso anche nel croato. ${ }^{16}$

Lo stesso discorso potrebbe probabilmente essere applicato anche al lessico del Krelj e in particolare all'uso che fa dei prestiti soprattutto romanzi. Nel suo recente saggio $\mathrm{M}$. Orožen ${ }^{17}$ sottolinea l'influsso esercitato su questo autore protestante dall'ambiente culturale in cui si formò. Fin dal secolo XI la valle del Vipacco si trovò nella sfera d'influsso del Patriarcato d'Aquileia ovvero dello Arcidiaconato friulano inferiore. E nelle vicine diocesi di Trieste e Capodistria operavano imperterriti i glagoljaši che tramandavano la liturgia paleoslava sotto il manto ufficiale del latino liturgico. Questo sottile influsso dei glagoljaši croati è percettibile così nelle opere del Krelj come lo sarà una cinquantina d'anni più tardi nel singolare Vocabolario Italiano-Schiavo di fra' Gregorio Alasia da Sommaripa del 1607 che operò da servita piemontese nella vicina Duino. ${ }^{18}$ Inoltre, come negli altri ambiti culturali, anche in quello del Krelj era notevole l'influsso linguistico del latino classico (nei rapporti con le autorità ecclesiastiche e con gli umanisti e studiosi), del tedesco letterario (lingua base dell' insegnamento e idee protestanti) e infine di tutte le lingue dei popoli che convivevano sotto l'Austria. In particolare va qui sottolineato l'influsso esercitato dalla lingua ceca e va ricordato che la stessa $O B$ del Krelj dimostra determinate affinità con il catechismo ceco di J. Jirke. ${ }^{19}$

In questa classificazione dei romanismi nel Krelj sono state prese in considerazione, oltre alla $O B$ e alla $P S, 15$ altre opere, finore censite, pubblicate prima delle sue e anche contemporaneamente. L'autore di tutte è naturalmente Primož Trubar. Parlando di censimento ci riferiamo agli utillissimi Alfabetariji che sta pubblicando l'Accademia delle Scienze di Lubiana. ${ }^{20} \mathrm{Gli}$ Alfabetariji sono degli accuratissimi re-

15 Si cfr. N. Godini, La fortuna dei friulanismi nello sloveno carsico mediano in "Ce fastu?" LVII (Udine 1981), pp. 93-108 e della stessa Sulla penetrazione dei prestiti romanzi nello sloveno in "Linguistica“ XXIV (Ljubljana 1984), pp. 303-313.

16. L'ipotesi di un dialetto misto è riportata ma non condivisa da J. Rigler, Kreljev jezik, op. cit., p. 140. Nelle scelte operate dal Krelj, il Rigler ravvisa piuttosto un tentativo di avvicinamento al croato, soprattutto dove gli esiti fonetici del croato coincidono con quelli dei dialetti carsici.

17 Cfr. M. Orožen, Kreljev jezikovni koncept, op. cit., pp. 20-21.

18 Si veda la bella riedizione del 1979 (I ubiana-Duino Aurisina-Trieste) che comprende anche i saggi Gregorio Alasia da Sommaripa e il suo vocaholario di L. L cgiša (pp. 228-285) e La nuova edizione di Alasia di B. Gerlanc (pp. 286-303).

19 Cfr. V. Burian, Po stopách čésství a češké knihy v starším slovinském pisemnictví in "Slavia" VIII (Praga 1929-1930), pp. 54-75, 248-270, 449-482 e in particolare pp. 248-252. L'opera di J. Jirke è Catechismus der Rechtgleubigen Boehemischen Brüder del 1554. Per inciso, a Jena il Krelj ebbe come compagni di studi numerosi studenti cechi e slovacchi e attraverso loro conobbe certamente le opere liturgiche che dal 1457 tramandavano l'insegnamento hussita.

20 Un grazie di cuore a tutta l'équipe della sezione "Historični slovarji“ dell'Accademia delle Scienze di I.ubiana. Mi è sempre stata di preziosissimo aiuto. Un grazie particolare a Francka Premk e France Novak. 
gistri del lessico di ogni singola opera degli autori protestanti sloveni, accompagnati da un esauriente saggio sui sistemi seguiti nella trascrizione del materiale, delle varianti, doppioni ecc. Il tutto in vista della pubblicazione dell'attesissimo dizionario storico della lingua slovena. Per citare un solo esempio, l'Alfabetarij della Otročja biblija del Krelj contiene 1327 lemmi di cui 1292 sono lessemi, 33 gli indicatori e 2 le direttrici.

Le 15 opere in questione sono: Abecedarium (apparso anonimo con lo pseudonimo "Philopatridus Illiricus") del 1550, Catechismus del 1550, Abecedarium del 1555, Catechismus del 1555, Ena molitov tih kerščenikov del 1555 (il testo sloveno è del Trubar, ma la firma è di P. P. Vergerio), Ta evangeli svetiga Mathevsha del 1555. Ta slovenski kolendar del 1557, Ta pervi del tiga noviga testamenta (1557-1558) ovvero En Regishter (1558) chiamato anche Mala postila (La postilla piccola), Svetiga Pavla ta dva listy del 1561, Articuli oli deili (1562), Cerkovna ordninga (1564), Abecedarium oli tabliza (1566), Ta celi catechismus (1567), Ena duhovska pejsem zuper Turke (1567) e infine Eni psalmi (1567). ${ }^{21}$.

$\mathrm{Ci}$ è sembrato opportuno scindere i termini dotti internazionali da quelli che possono essere considerati dei veri e propri romanismi. La loro base può essere greca oppure latina, ma spesso essi arrivano nel lessico sloveno comune attraverso la mediazione germanica. Questo primo gruppo comprende: aloa 'aloe', altar, oggi oltar 'altare', angel 'angelo', antikrist 'anticristo', apostol 'apostolo', con il derivato apostolski 'apostolico' (agg.), ceremonija 'cerimonia', dekret 'decreto', denar 'denaro', eksempel 'esempio' (oggi considerato antiquato, SSKJ I, 55722, element 'elemento', episkop 'vescovo' (oggi 'vescovo' nella chiesa orientale, SSKJ I, 597), epistola 'epistola' (SSKJ I, 597), evangelij 'Vangelo' (SSKJ I, 605-6), evangelion 'idem' (assente nello SSKJ), evangelist 'evangelista', farizej 'fariseo', kalender oggi koledar 'calendario', kapitul 'capitolo' oggi kapitelj (SSKJ II, 280), kardinal 'cardinale', katekizem 'catechismo', katoliški 'cattolico', (agg.), koncilij 'concilio' oggi koncil, komet(a) 'cometa', kristjan 'cristiano', kristjanski 'cristiano' (agg.) oggi considerato antiquato e sostituito da krščanski (SSKJ II, 491), križ 'croce', mana 'manna', papagaj 'pappagallo', papist 'papista, fautore del papato', parabola 'parabola', paradiž 'paradiso', pogan 'pagano', postila 'postilla', prelat 'prelato', pridiga 'predica', purpur 'porpora', oggi considerato letterario (SSKJ IV, 290), regula 'regola', oggi soltanto nell'aggettivo regularen 'regolare' e regularnost 'regularità' (SSKJ IV, 463), sentenc(ij)a 'sentenza', oggi sentenca (SSKJ IV, 629), sigill 'sigillo', presente solo nel Krelj in PS con la frequenza 8, oggi in disuso, sofist 'sofista', statut 'statuto' con la variante štatut come del resto oggi, testament 'testamento', vigilija 'vigilia' e infine zakrament 'sacramento'.

21 Per una dettagliata descrizione delle opere di quel periodo si veda soprattutto Abhandlungen über die slowenische Reformation. I. Band (Trofenik, München 1968).

22 SSKJ sta per Slovar slovenskega knjižnega jezika I/A-H (Ljubljana 1970), II/I-Na (Ljubljana 1975), III/Ne-Pren (Ljubljana 1979), IV/Preo-S (Ljubljana 1985). 
In alcuni di questi prestiti è evidente la mediazione germanica che resta comunque difficile da quantificare. Del resto, la mediazione da parte delle lingue germaniche così dei termini romanzi come dei termini internazionali resta un problema difficilmente risolvibile nel complesso processo di formazione e storia della lingua slovena letteraria.

Nel caso specifico dei romanismi nelle opere del Krelj va anche tenuto in evidenza che non è un dato sconto che il Krelj utilizzasse tutte le opere del Trubar pubblicate prima delle sue. Inoltre non è da scartare la probabilità che alcuni dei romanismi presenti nelle opere di tutti e due fossero già entrati nell'accettazione linguistica comune. In questo caso i due autori protestanti ne sarebbero stati soltanto $i$ codificatori.

Tenendo presente queste necessarie cautele elenchiamo nel secondo gruppo i romanismi presenti nel Krelj e, rispetto al primo gruppo, non molto frequenti nelle opere del Trubar che ci servono da confronto. Sono questi dei romanismi veri e propri e soltanto alcuni possono far sospettare anche la solita mediazione da parte delle lingue germaniche. Li accompagna una sintesi etimologica.

(Per le abbrevazioni usate si veda la nota $\mathrm{n} .23$ )

bandero 'vessillo, bandiera“. Termine dotto internazionale dall'ital. bandiera (Bezlaj I, 11, Mende 42);

falš 'falso' (agg.) Dal lat. falsus, ital. falso, da notare però che nelle zone del nord jugoslavo subisce la mediazione del ted. falsch (Skok I, 504);

form(a) 'forma'. Latinismo oppure italianismo, documentato fin dal 15. secolo (Skok I, 525-6);

fundament 'fondamento'. Dall'ital. fondamento (Skok I, 523 s. v. fond);

goljufati 'truffare, ingannare' e goljufija 'truffa, ingano'. Dall'ital. gaglioffo 'ribaldo, manigoldo' (Skok I, 548 s. v. galuf che lo considera un prestito antico per il passaggio della $a$ a $o$; Bezlaj I, 159; Mende 70 goljuf 'imbroglione' dall'ats. galufá 'rubar';

23 Le abbrevazioni usate:

Bezlaj = F. Bezlaj, Etimološki slovar slovenskega jezika I/A-J (Ljubljana 1977), II/K-O (Ljubljana 1982).

Boerio = G. Boerio, Dizionario del dialetto veneziano (Venezia 1856).

DEI = C. Battisti. G. Alessio, Dizionario etimologico italiano I-V (Firenze 1975).

Doria = M. Doria, Grande dizionario del dialetto triestino (storico, etimologico, fraseologico (con la collaborazione di C. Noliani) Ed. Il Meridiano, Trieste 1987.

Mende = Mende, Romanische Lehnwörter im Slowenischen. Inaugural-Dissertation zur Erlangung des Doktorgrades. (Edizione dattiloscritta in "Slavisches Seminar der Freien Universität Berlin"), 1951.

Pirona = G. A. Pirona, E. Carletti, G. B. Corgnali, Il nuovo Pirona. Vocabolario friulano (Udine 1977).

Skok = P. Skok, Etimologijski rječnik hrvatskoga ili srpskoga jezika I-IV (Zagreb 1971-1974).

Štrekelj, DAW = K. Strckelj, Zur slavischen Lehnwörterkunde (DAW L 3) (Wien 1904).

Sturm $=\mathrm{F}$. Śturm, Refleksi romanskih palataliziranih konzonantov v slovenskih izposojenkah in “Č.IKZ“" VI (I.jubliana 1927), pp. 45-85. 
idioma 'idioma'; linguaggio proprio o particolare di un popolo, nazione o regione. Attraverso il lat. idioma dalla base greca (DEI III, 1916);

infula 'infula, fascia bianca con cui il sacerdote pagano si cingeva il capo' (DEI III, 2024), dal lat. infula;

insula 'isola'. Chiaro latinismo, Trubar riporta inzula;

kapa 'berretto'. Dal lat. cappa, ma documentato in quasi tutte le lingue europee. Per lo sloveno, ceco e slovacco si suppone però la mediazione germanica (Bezlaj II, 17);

kapelica 'piccola cappella, chiesetta'. Dal lat. capella 'mantello' (il mantello di s. Martino nella cappella reale francese), poi 'cappella'. Kapelica ne è il diminutivo (Bezlaj II, 17);

kaštigan 'castigato' e kaštigati 'castigare'. Dall'ital. castigare e castigo (Mende 78) anche se nello sloveno e nel croato-kajkavo è possibile la mediazione dell'aated. chastigon, a sua volta dal lat. castigare (Skok II, 58 s. v. kastig).

levit 'levita, appartenente alla tribù ebraica di Levi'. Termine dotto internazionale; ljuljka 'loglio, della famiglia delle graminacee'. Dal lat. lolium, probabilmente con la mediazione dell'ital. (Bezlaj II, 146). Però come già lo Skok (II, 330 s. v. lûlj) anch'egli suppone una lontana origine mediterranea del termine;

morija 'moria, grande mortalità di uomini o di animali per contagio'. Skok (II, 457 s. v. mortòrij) parte dal lat. morior e la considera un postverbale in -ija da moriti 'uccidere'. Bezlaj (II, 195) risale al psl. + moriti, + morjon 'uccidere, io uccido'; mutast 'muto' (agg.). Lat. mutus, ital. muto, presente anche nel scr. (Skok II, 491-2 s. v. muta). Da notare il suff. autonomo -ast;

natura 'natura', oggi considerato solamente letterario (SSKJ II, 1012-3) e naturski 'naturale', oggi sostituito da naturen, anch'esso poco usato. Dal lat. o ital. natura; olje 'olio'. Si parte dal greco da una ignota fonte mediterranea, ma è il lat. oleum, lat. volg. oliu a mediarlo in quasi tutte le lingue europee (Bezlaj II, 247). Skok (II, 543 s. v. ulje) parte dal nom. pl. lat. olea;

oljka 'ulivo e oliva' (albero e frutto), come già nel lat. oliva. ital. oliva (Bezlaj, II, 247 e Skok III, 543);

oštarija 'osteria'. Ital. osteria, ven. ostaria, friul. ostarie (Mende 100 e Bezlaj II, 260);

pena 'pena, danno fisico o morale a chi è oppure è ritenuto colpevole di qualcosa' Dall'ital. pena che deriva dal lat. poena (Skok II, 635);

persona 'persona, individuo'. Dall'ital. ovvero lat. persona (Skok II, 643);

procesion 'processione'. Dall'ital. processione (Skok III, 48 s. v. proces). La variante procesija si è mantenuta;

regula 'regola, norma'. Dall'ital. ovvero lat. règula, voce dotta internazionale (DEI V., 3224 e Skok III, 125);

špendija 'dispendio': secondo lo Skok (III, $636 \mathrm{~s}$. v. pendžati) in sloveno è un prestito dall'ital. dispendio. Probabile la mediazione dell'ital. spendere, a sua volta dal lat. expendere (DEI 3584) o meglio dal ven. ts. spender. Troviamo inoltre špendati 'spendere', e špendanje 'lo spendere; spreco';

štima 'stima, apprezzamento' e štimati 'apprezzare'. Dallo ital. stima e stimare, dal lat. aestimare (DEI 3636, Mende 115 e Skok III, 335); 
trta 'vite'. Dal lat. torta, part. pass. femm. di torquère secondo Štrekelj (DAW 50, p. 67-68) e Skok (III, 511). In ital. ant. c'è torta 'curvatura, piegatura', con il secondo significato 'fascio, legame', si veda DEI 3835.

Il terzo gruppo vede allineati i romanismi presenti soltanto nelle opere del Krelj. Essi non trovano riscontro nelle opere del Trubar, siano esse antecedenti o contemporanee, quelle di cui ci siamo valsi per quest'analisi comparata.

Veri e propri romanismi kreljani sono dunque:

barkador 'barcaiolo'. Il nome base barka con il derivato barkarol è presente solo in un'opera del Trubar (Ta pervi del... 1557-8). Barka è unanimemente considerato di origine mediterranea (DEI 436 e Skok I, 113). Barkador non trova però riscontro in nessuna variante dei dialetti vicini, così ital. o croati, dove è invece presente piuttosto barcarol oppure barcaiol. Si veda anche Doria 56-57;

beč 'denaro spicciolo, moneta', presente anche nel scr. Dal ven. bezzo 'antica moneta veneta coniata dal 1497 in poi' (DEI 50 s. v. bezzo) con il pl. bezzi 'monete, soldi' (Bezlaj I, 14). Doria 69 considera il termine bezo 'soldo, quattrino, moneta di rame equivalente a sei denari' comune a tutto il Veneto e Ven.-Istr., presente anche nell'ant. friul. bêc oppure bêz 'moneta veneziana di rame del valore di mezzo soldo' e come già il DEI lo fa risalire al ted. svizz. Bätze (mated. Betz) 'moneta piccola'. Cfr. anche Mende 44 e Skok I, 130 s. v. beči;

butega 'bottega, negozio di commestibili'. Doria $87-88$ come già DEI $576 \mathrm{~s}$. v. botega rileva che il termine è panromanzo, escluso il rumeno. La base è il greco apothèkè 'ripostiglio', lat. apothéca 'magazzino, luogo dove si teneva il vino nella parte alta della casa'. Ven. ts. botega (Skok I, 245 s. v; butiga, Strekelj, DAW 50, Šturm, ČJKZ VI, 58). Per la presenza della $u$ consideriamo butega piuttosto un prestito dal friul. buteghe (Pirona 86);

celibat 'celibato', voce dotta internazionale. Dal lat. caelebs, -ibis, di etimologia sconosciuta, forse prestito o relitto del sostrato mediterraneo, franc. célibat nel XVI sec. (DEI 844 s. v. celibe);

cetha oppure cetka 'ittionimo'. Cetka può considerarsi il dim. di ceta, ittionimo che il Radnić registra nel 17. sec. (Skok I, 85-6 s. v. kit 'balena'). Per DEI 880 ceta e 881 ceto è voce dotta dal lat. cetus, a sua volta dal gr. kêtos 'mostro marino, in particolare la foca, più tardi cetaceo in genere; balena'. Di etimologia sconosciuta;

dekret 'risoluzione promulgata con editto, decreto, deliberazione' (DEI 1229 e Skok I, 389), dal lat. decretum;

fundati se 'sprofondare, distruggersi, perire'. DEI 77, Skok I, 53 e da ultimo Bezlaj $(\mathrm{I}, 133)$ che lo considera in prestito dall'ital. affondare, comunque panromanzo e presente anche nel scr. kaj., si veda il lat. affundere;

husit '(h)ussita, seguace di Jan Hus“, voce dotta internazionale;

irati se 'adirarsi, arrabbiarsi'. Dal lat. ira ovvero dall'ital. antico irare 'adirare' (DEI 2103). Da notare la forma riflessiva che è autonoma;

izop 'isopo oppure issopo', Hyssopus officinalis, voce dotta inter. (DEI 2117);

križma 'unguento, olio santo'. Può avere le varianti krizma e hrisma e oltre che nel 
scr. (Skok II, 196-7) è conosciuta anche in ceco, polacco e ucraino. Dal greco ovvero lat. chrisma (Bezlaj II, 94 come già DEI 1154 e Skok II, 196-7);

krlj 'corlo, ceppo, tronco' che secondo Strekelj (JA XII, 460) è un prestito dall'ital. ven. corlo 'toppo, pezzo di legno grosso e talvolta informe, nel quale sta ficcato il fuso, e serve di piedestallo o base dell'arcolaio'. Boerio 199 e Doria 176. Skok (II, 203-4) suppone che il ven. corlo possa essere lo stesso corlo 'rullo' dal lat. + currulus, dim. di currus 'carro' (DEI 1112). Il sinonimo di krlj è però in scr. krklja. 'caudex' e questa etimologia rende inspiegabile la $k$. Bezlaj (II, 95) si dichiara perplesso e risale al psl. $+k \hat{b} r$-j6;

mandrija 'pecorile, ovile'. Dal gr. e lat. mandra 'ovile chiuso', ital. mandria 'ovile, branco di bestiame, quantità di bestie', ven. mandra (Boerio 392), friul. mandre, mandrie (Pirona 560/61; (DEI 2343 lo considera un probabile relitto del sostrato mediterraneo, cfr. anche Skok II, 368 s. v. mandra e Bezlaj II, 165);

mediator 'mediatore'. Voce dotta inter. dal lat. mediare 'essere intermedio' (DEI 2402);

moneta 'moneta'. Chiaro latinismo o italianismo dal lat. moneta (DEI 2496 e Skok II, 454);

palacij 'palazzo', Trubar: palač. Dal tardo lat. palatium (DEI 2728) oppure relitto della latinità balcanica, dal nom. pl. palatia, poichè in slov. diventerà paláča e scr. pàlača (Skok II, 590);

palm(a) 'palma'. Dal lat. o ital. palma (DEI 2734 e Skok II, 594). Nella PS, si alternano il m. e il femm.;

patron 'padrone, chi può agire a suo arbitrio'. Dal lat. patronus, relitto lessicale dalmata-romanzo (Skok II, 623 s. v. patrun, DEI 2718-9);

poet 'poeta', dal lat. poeta, voce dotta (DEI 2968);

policija 'governo'. Voce dotta, dal tardo lat. polıtıa ovvero gr. politéia 'arte di governare i cittadini'. Nel senso moderno di 'polizia' è un francesismo diffuso dalla Rivoluzione (DEI 3002); così anche Skok II, 698;

prebenda 'prebenda, diritto di un ecclesiastico di fruire della dote annessa al súo ufficio'. Voce dotta dal lat. praebenda, n. pl. 'cose da fornire' (DEI 3051 e Skok III, 49 s. v. profont);

publikan 'publicano, gabelliere, daziere'. Voce dotta dal lat. publicanus, agg. lat. in -anus da publicum (DEI 3138 e Skok III, 65 s. v. publik);

sorta 'specie, forma, qualità'. Dall'ital. sorta oppure sorte (DEI 3560 e Skok III, 307);

štentati se 'far fatica, incontrare difficoltà'. Italianismo da stentare.(DEI 3629 'aver scarsità, indugiare' e Skok III, 413); ven. ts. stentar (Boerio 702);

termin' 'limite, termine'. Dal lat. terminus oppure termen, -inis, dapprima 'pallo di confine', poi 'limite, termine', panromanzo (DEI 3760). Skok (III, 461 s. v. têrmen) propende per l'ital.;

užanca 'usanza, consuetudine, costume'. Dall'ital. usanza che è un astratto lat. in -antia da usare, diffuso anche nell'istriano (Skok III, 554, s. v. užati). Cfr. anche DEI 3962. Dall'ital. usura deriva lo slov. ožura 'interessi da pagare' che svilupperebbe poi secondo Bezlaj (II, 265), secondariamente nei dialetti slov. e scr. il significato 'costume, consuetudine, usanza'. 
Prispevek povzema glavno problematiko, ki se pojavlja v delih Sebastijana ali Boštjana Krelja. Protestantski avtor se nam je namreč zdel deležen premajhne pozornosti in nekoliko zapostavljen $\mathrm{v}$ teh zadnjih letih, ki so polna proslav in obletnic reformacijske epopeje.

$\mathrm{V}$ naših literarnih zgodovinah se od vselej ponavlja trditev, da je Krelj vnesel v tkivo slovenskega jezika številne romanske izposojenke, ker je pač doma z obrobnega območja slovenskega življa, ki je izpostavljen vplivom romanskih sosedov in tudi zato, ker naj bi hotel zavreti pretirano uporabo germanskih izposojenk, ki so značilne za ostale protestantske pisce.

Pretres romanizmov, ki so prisotni v 15 Trubarjevih delih in v glavnih dveh Kreljevih, Otročji bibliji in Postili slovenski, pa dokazuje, da niso izrecno Kreljevi romanizmi tako zelo številni. Precej je namreč romanskih izposojenk, ki so že prisotne $v$ Trubarjevih delih, tako starejših kot sočasnih. Seveda pa ne smemo pri tem prezreti možnosti, da je njihova prisotnost $v$ delih obeh protestantskih avtorjev lahko tudi slučajna. 